\title{
Cellular network Marine Sensor Buoy
}

\author{
Alexander Przybysz, Carlos M. Duarte,Nathan R. Geraldi, Jurgen Kosel, Michael L. Berumen \\ CEMSE, RSRC, CBRC, BESE \\ King Abdullah University of Science and Technology \\ Thuwal, Saudi Arabia \\ Email: alexander.przybysz@kaust.edu.sa,jurgen.kosel@kaust.edu.sa,
}

\begin{abstract}
Studies in the marine environment require devices to gather data in remote locations. These devices commonly use some form of data logging that require the user to retrieve the device after a period of time. This is not only a large effort, but often presents an unnecessary delay and risk in the event that the device malfunctions, goes missing or if the data collected is redundant. We propose a modular, remote and autonomous sensing solution that enables multi-sensor readout and wireless data communication, providing scientists with real time data. This sensor buoy is comprised of a primary module which is a data logger that connects to an online server via mobile network. The secondary portion is the hub that connects to an array of sensors that are customized to the needs of the marine scientists.
\end{abstract}

Keywords - remote, sensing, modular, marine, real time

\section{INTRODUCTION}

The study of marine environments remains a highly researched field, although a vast portion of the Earth's aquatic lifeforms have yet to be discovered [1]. In these days and age it has become important to investigate the impact of humans on aquatic life, including carbon dioxide emissions, noise and hydrocarbon spills[2]. Researchers in marine environments often face challenges such as weather, accessibility and reliability of devices. Currently, many research efforts employ the use of research vessels to mitigate these challenges. These vessels require a substantial crew and resources to conduct experimental work over extended periods of time, which makes them an expensive solution to even the smallest of research tasks. Driven by the need for fast access to data and wireless capability, the use of oceanic monitoring buoys has become a favorable alternative. Universidad Politecnica de Valencia proposed a buoy that collects salinity, temperature, hydrocarbon contamination and wind data remotely [3]. The buoy uses $\mathrm{Wi}-\mathrm{Fi}$ to transmit data which has high data rates and removes the need for wires. However, the transmission range is limited to approximately $400 \mathrm{~m}$ line-of-sight, based on the transceiver used in the buoy.

Marine research efforts are not limited to investigating the impact of humans on the oceans but also study the triggers of catastrophic events that may have damaging impacts on humans. The early detection of seismic events and tsunamis offshore have played a crucial role in emergency response protocols. Such instrumentation has been implemented of the coast of Japan and has been used to understand the generation and directional propagation of tsunamis in greater detail. Tsunami probes detect seismic shock or irregular disturbances in the water column through the use of accelerometers, hydrophones and differential pressure sensors on the seabed[4]. These sensors are connected by long distance cables to a coastal base station. The Ocean University of China has developed a buoy that communicates with sea bed sensors using acoustic transceivers, which in turn relays the data via Beidou Satellite to a server [5]. The use of satellites enables wireless communication with the buoy anywhere in the world. The maximum communication distance using acoustic methods is $1,500 \mathrm{~m}$, with a data transfer rate of $2 \mathrm{~kb} / \mathrm{s}$. This allows for the real-time sampling of sensor data at a rate of $5 \mathrm{~Hz}$ for a 50 character string. Although the use of satellites allows for worldwide access to sensor data, this method of communication suffers from low data transfer rates at $77 \mathrm{~B} / \mathrm{s}$, and transfer costs as high as 900 GBP/MB for the Iridium network [6]. As a robust, passive and cost-effective solution for monitoring biodiversity on reefs, the Autonomous Reef Monitoring System (ARMS) has recently been developed. ARMS is an array of PVC shelves that is fastened to a reef, allowing micro-fauna to colonize the structure [7]. Subsequently, ARMS units are manually retrieved to analyse the micro-fauna.

Existing techniques and standard equipment are not robust in their capability or flexibility to incorporate various sensor types. This limitation prevents researchers from taking advantage of modern developments in sensor technologies[8$12]$.

The proposed solution is a marine buoy that provides novel capabilities achieved through the implementation of GPRS based communication techniques and multi-sensor capabilities. It allows for sufficient data transfer rates whilst maintaining remote capability at distances up to the limits of the network service, which can be anywhere from 2 to $35 \mathrm{~km}$ [13]. An 8-channel digital serial and analog hub allows for connection to a wide range of sensors. 


\section{METHOD}

\section{A. Concept}

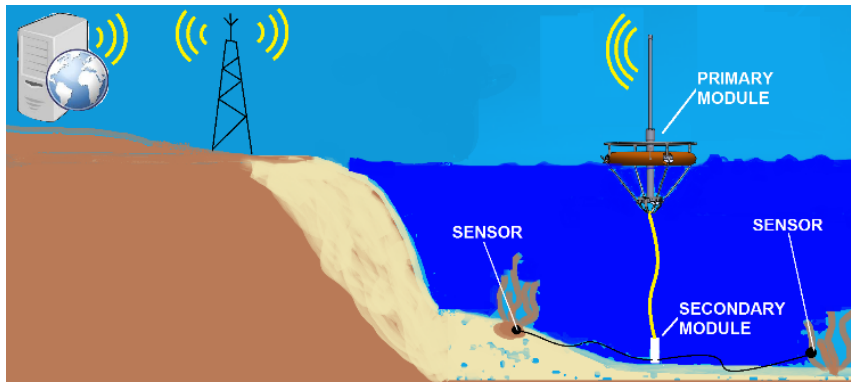

Figure 1: Concept of multipurpose sensor buoy.

The proposed solution is a buoy that houses a data logger and transmitter, as shown in Figure 1. The primary module is located in the buoy and is connected to a secondary module on the seabed via cable. The secondary module is a hub that can support a variety of sensors.

\section{B. Hardware}

\section{1) Primary Module}

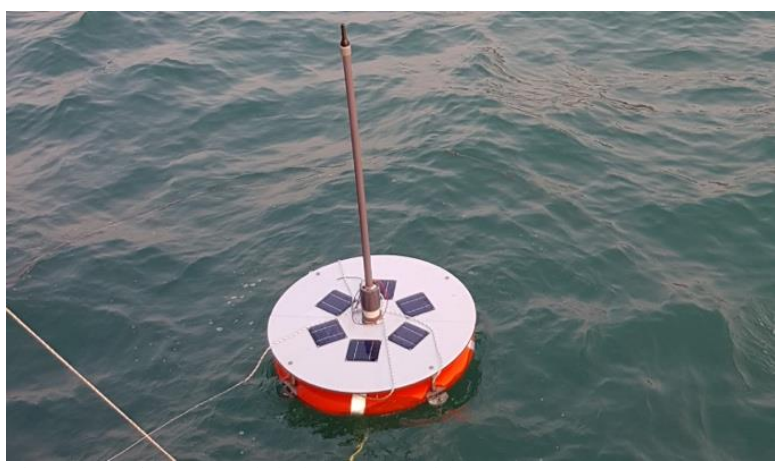

Figure 2: Primary module buoy assembly.

The primary module is housed in a buoy, shown in Figure 2. The module makes use of a microcontroller (Espressif Systems, ESP32) connected to three modules, namely; a GPRS evaluation board (SIMCom, SIM800L), a Micro-SD card module, a Real Time Clock module (Maxim Integrated, DS3231) and resetting circuit, as shown in Figure 3. This module periodically sends read commands to a secondary module via the Universal Asynchronous Receiver/Transmitter (UART) bus. In a sense, the primary module is a master device and the secondary module is the slave.

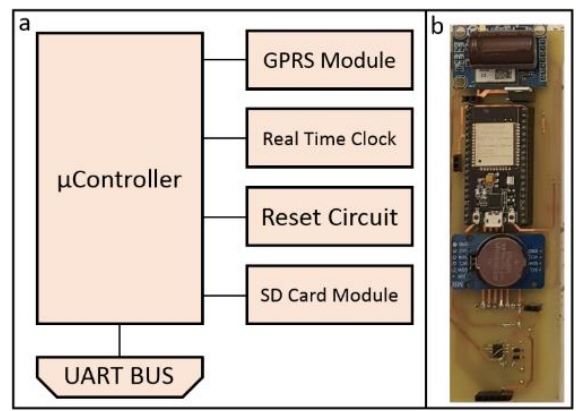

Figure 3: Primary module block diagram (a) with primary module circuit (b).
The software for the primary module is kept as simple as possible in order to ensure maximum reliability. The primary module, on power up, begins with an initialization sequence, which focusus on securing a connection with the GPRS network. In the event that the connection times out or fails for some reason, the primary module resets itself. It will not proceed if a connection is not established. Figure 4 shows the program of the primary module.

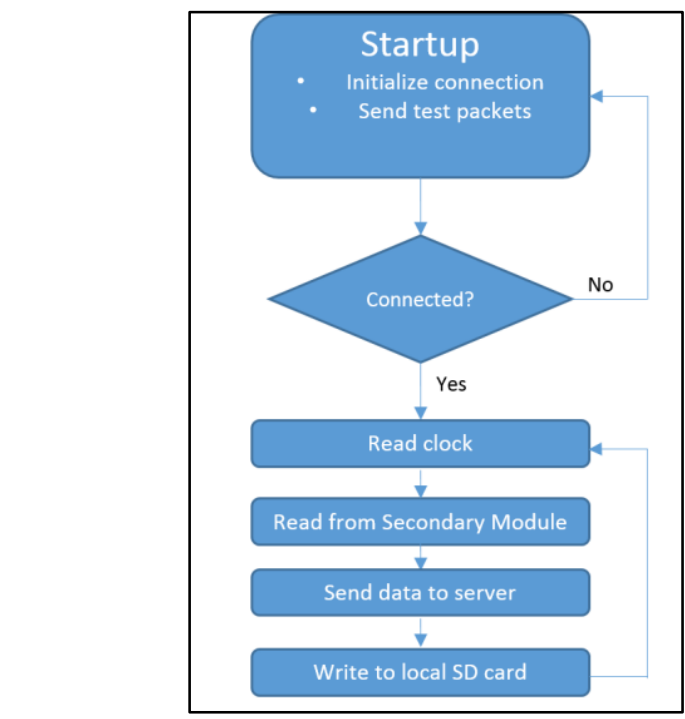

Figure 4: Program flow of the primary module.

\section{2) Communication}

The communication module used is a SIM800L evaluation board. This evaluation board is interfaced with the microcontroller using UART protocol at 19,200 bauds using AT commands that are sent with a carriage return character.

Alternative infrastructures that have been used for communication are LoRa, Sigfox, Wi-Fi and WiMax. The main metrics of concern are range, cost and throughput. LoRa and Sigfox perform similarly with the main difference being the bandwidth. Sigfox has a narrow bandwidth of $100 \mathrm{~Hz}$ and is designed for low sampling rates, LoRa has a bandwidth of $125 \mathrm{kHz}$ which enables a higher data rate and GPRS has a slightly higher bandwidth of $200 \mathrm{kHz}$ [14]. Wi-Fi has high throughput but performs poorly in range, on the other hand WiMax can provide a slightly higher throughput with an extended range that may transmit up to $35 \mathrm{~km}$ [13]. The application of WiMax is for creating wireless internet pointto-point links which incorporates fixed directional antennas, which would be a disadvantage for a device that is exposed to constant wave motion.

Given the aforementioned metrics, the only viable options are LoRa and GPRS, which have similar bandwidths with the main difference being infrastructure. For the time being, GPRS has a built-in infrastructure, whereas LoRa requires a base station/gateway as close to the LoRa transmitter as possible. Therefore, we chose GPRS for this application.

The connection to the server is performed using a GPRS connection to an online server using a prepaid data package, 
which can be recharged remotely. The connection to the server is made using TCP/IP protocol.

\section{3) Energy Management}

The power supply of the buoy is comprised of three parts; a solar cell array, a battery charger and a battery, as shown in Figure 5 . The solar cell array is made up of six cells $(1.25 \mathrm{~W}$, $5 \mathrm{~V}, 250 \mathrm{~mA}$ ), totalling to a maxiumum power of $7.5 \mathrm{~W}$. The battery is made up of 30 Lithium Ion cells (3.7 V, $4.2 \mathrm{Ah})$ giving a total capacity of $126 \mathrm{Ah}$ or $466.2 \mathrm{Wh}$. This voltage is then stepped up using a converter to approximately $5 \mathrm{~V}$ (Aerosemi, MT3608). The theoretical lifetime of the battery given a measured average power consumption of $1.173 \mathrm{~W}$ is 397 hours.

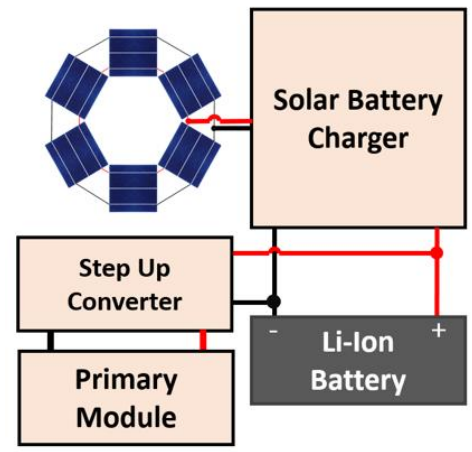

Figure 5: Energy management system of primary module.

The charger used is a dedicated Lithium Ion cell charger (Adafruit Industries, MCP73871) capable of delivering a maximum charging current of $1 \mathrm{~A}$. The solar battery charger is primarily dependent on the supply voltage and uses a method called Votage Proportional Charge Control (VPCC) which limits the current drawn from the solar cells in order to maintain the charging preset voltage [15].

The theoretical lifetime of the system as whole is evaluated for the shortest day of the year, winter solstice, which is considered for the worst case scenario. The wather conditions are assumed to be clear. The maximum power from the solar cells is multiplied by the Sine of the altitude angle of the sun. Figure 6 shows the power vs time graph for winter solstice for the latitude of Jeddah, Saudi Arabia. The red area is the energy used by the device and the blue area is the energy gained by the solar cells. Provided there are no clouds the energy gained for the 24 hour cycle is $30.11 \mathrm{Wh}$ and the enrgy loss is $28.15 \mathrm{Wh}$ leaving a $1.96 \mathrm{Wh}$ of energy.

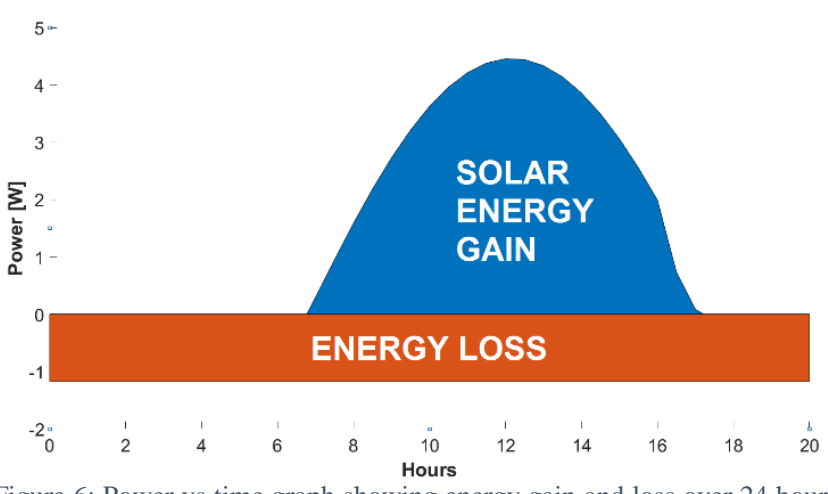

Figure 6: Power vs time graph showing energy gain and loss over 24 hours of winter solstice.

\section{4) Reset switching}

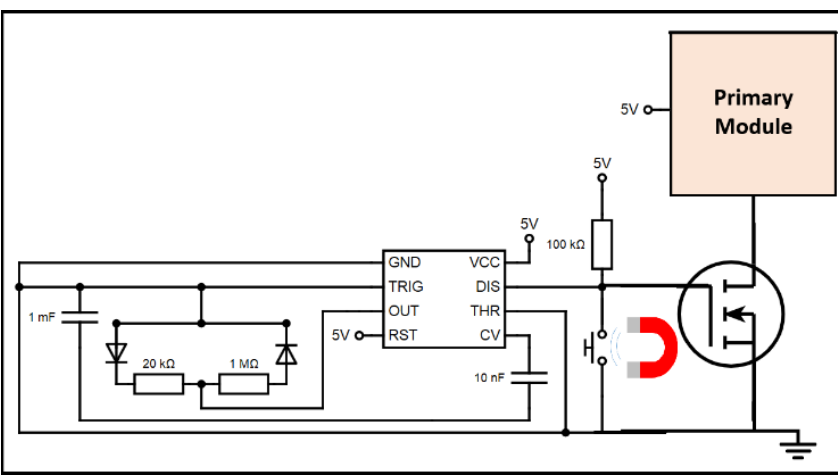

Figure 7: Resetting circuit for primary module.

The device is made such that the user does not have to open the housing to activate it. It can be kept dormant using a magnetic clip, which closes a normally open magnetic reed switch located on the primary module. This works alongside a master reset circuit to switch on an N-channel MOSFET. The circuit uses a Texas Instruments TLC555 timer in a high duty cycle long period design, as shown in Figure 7. The large capacitor and high resistance creates an astable oscillator with a period of 15 minutes. Since the duty cycle is $98 \%$ the offtime is 20 seconds. During this off- time the primary module is switched off to clear any possible errors that may have occurred. The magnetic reed switch bypasses the timer to always keep the MOSFET gate voltage low. When the user wants to deploy the buoy, the magnetic clip is removed and the timer circuit is left to do the resetting. In total the power consumption of the buoy during dormancy is approximately $1 \mathrm{~mW}$ at the supply voltage of $5 \mathrm{~V}$.

\section{5) Secondary module}

The secondary module is customized to the needs of the marine researcher and application. In the case presented herein, it is connected to Hall-effect sensors with integrated temperature sensors (Silicon Labs, Si7210) used to measure valve gaping of giant clams [16]. Analog blue light sensors (Pacific Silicon Sensor, PC10-2_TO5) are used to assess the level of incident light on giant clams [17]. The sensors are 
connected to a multiplexer, which is connected to an Arduino Pro Mini, as depicted in Figure 8. The sensor data is then relayed via the UART bus to the primary module. Other sensors can be added to measure the behaviour of the giant clams, and obviously for other monitoring applications, to ambient stressors such as salinity and ambient noise [18].

The multiplexer expands the analog input (I2C SDA) on the microcontroller to eight switchable channels. The inherent benefit of this is that it can alternatively be used to monitor eight analog devices or a combination of both analog and $\mathrm{I} 2 \mathrm{C}$ sensors.

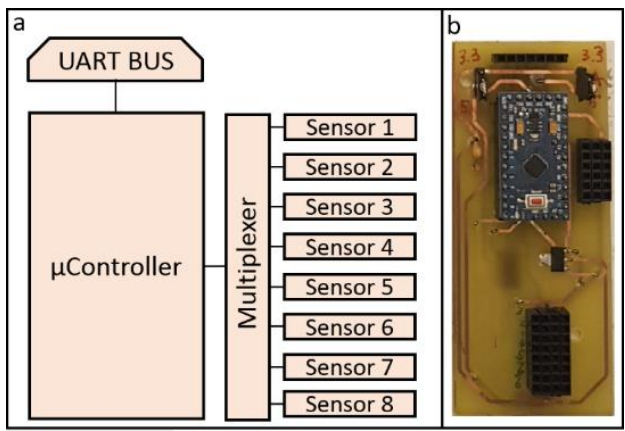

Figure 8: Secondary module block diagram (left) with image of secondary module circuit board (right).

Since these sensors are not inherently submersible in water, a packaging was fabricated to hermetically insulate and terminate the connections. This was done by creating a breakout board and placing it between two embossed profiles of $3 \mathrm{~mm}$ thick acrylic, as shown in Figure 9. Once the wires were terminated and the device was confirmed to be working the cavities were flooded by polyurethane epoxy. These sensors are placed on one jaw of a giant clam while a magnet is placed on the opposite jaw. The distance is determined from the proximity of the magnet to the sensor.

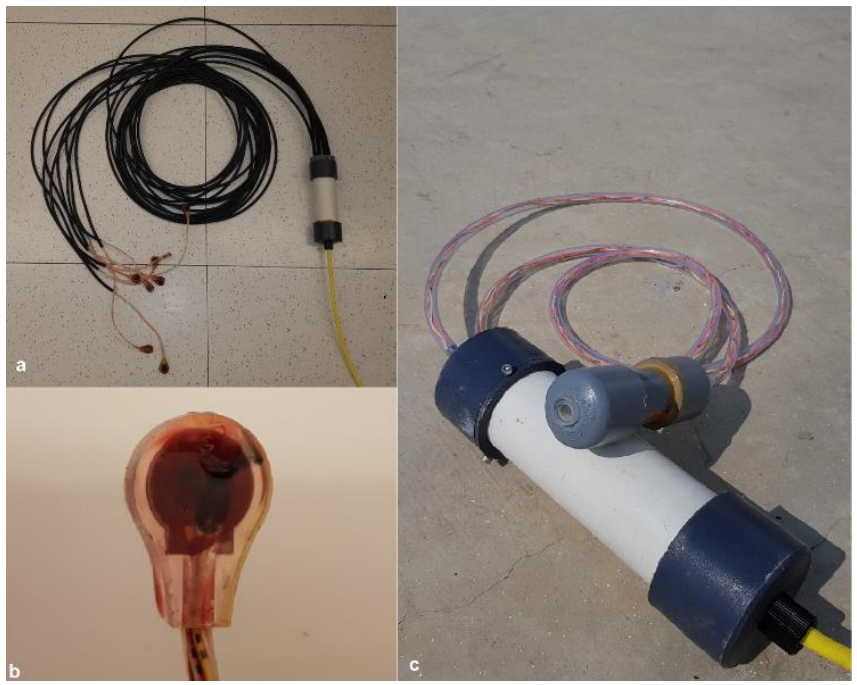

Figure 9: The secondary module enclosure with sensors (a). Encapsulated Hall-effect and temperature sensor (b).Secondary module with blue light sensor (c).

\section{RESULTS \& DISCUSSION}

Laboratory measurements were conducted with the magnetic field sensor for the purpose of measuring sensor response, data transmission rate and power consumption. The system as a whole was tested in the Red Sea around $100 \mathrm{~m}$ offshore with a light sensor 1 meter depth. Using a $30 \mathrm{~mm} \times 3 \mathrm{~mm}$ $\mathrm{NeFeB}$ disk, the response of the Hall effect sensor was measured with the magnet placed from $20 \mathrm{~mm}$ to $100 \mathrm{~mm}$ away from the sensor at $10 \mathrm{~mm}$ steps. The sensor characteristic curve is shown in Figure 10.

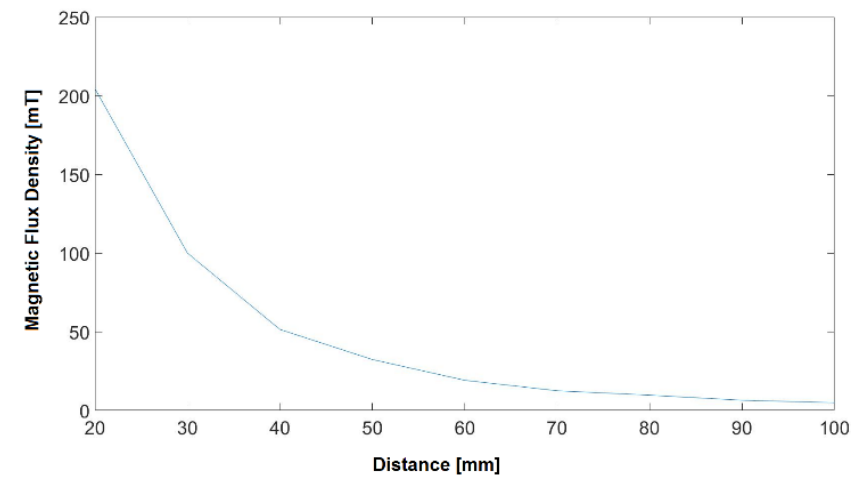

Figure 10: Characteristic curve of magnetic sensing system implemented by a Hall-effect sensor measuring the flux density of an $\mathrm{NdFeB}$ magnet as a function of distance.

The data transmission rate was measured by opening a TCP/IP port for listening and then fixing a delay between each transmission by $100 \mathrm{~ms}(10 \mathrm{~Hz})$. The primary module is capable of transmitting a 50 character string every $100 \mathrm{~ms}$, which results in a data rate of $4 \mathrm{kbps}$ to the server from where it is pipelined directly to a CSV file.

The power consumption of the unit as a whole was measured by using a $1 \Omega$ shunt resistor. The startup power consumption was neglected for this experiment. The current consumption at the $5.1 \mathrm{~V}$ end of the power supply was on average $230 \mathrm{~mA}$. Therefore, the average power consumption is $1.173 \mathrm{~W}$. This gives the unit a battery life of approximately 16 days without charging.

The measurement of the light sensor recording the intensity of blue sunlight over a period of 24 hours is shown in Figure 10. The data transmission rate was set to $4 \mathrm{~Hz}, 275,597$ samples were recorded. Figure 11 shows the light exposure level versus time. The maximum power output of the solar cells measured in direct sunlight at noon was $6.75 \mathrm{~W}$. Calibrating the light intensity profile with this maximum value results in the power profile, delivered by the solar panels, shown in Figure 12. The average power value from this profile is $4.73 \mathrm{~W}$ over a 12 hour daylight cycle (6:00 to 18:00). This leaves an average power of $3.557 \mathrm{~W}$ for charging the battery during the day. Provided the solar cells receive a minimum of 6 hours of sunlight per day, the whole system will run indefinitely. This does not take into account the degradation of cells from repetitive charging. 


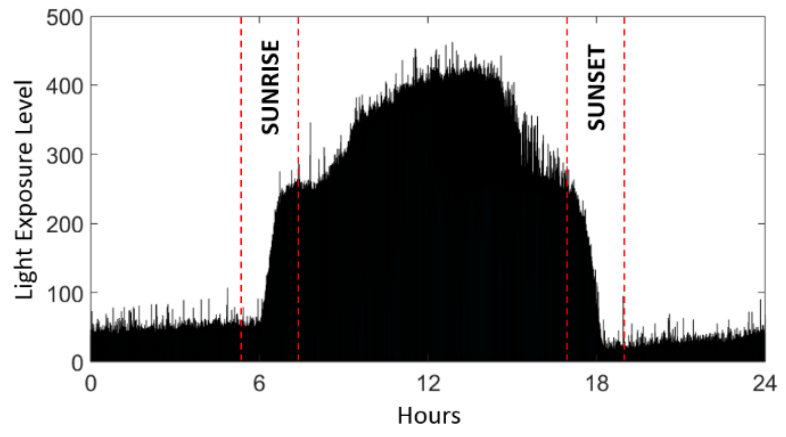

Figure 11: Light exposure level over a 24 hour period recorded in the Red Sea.

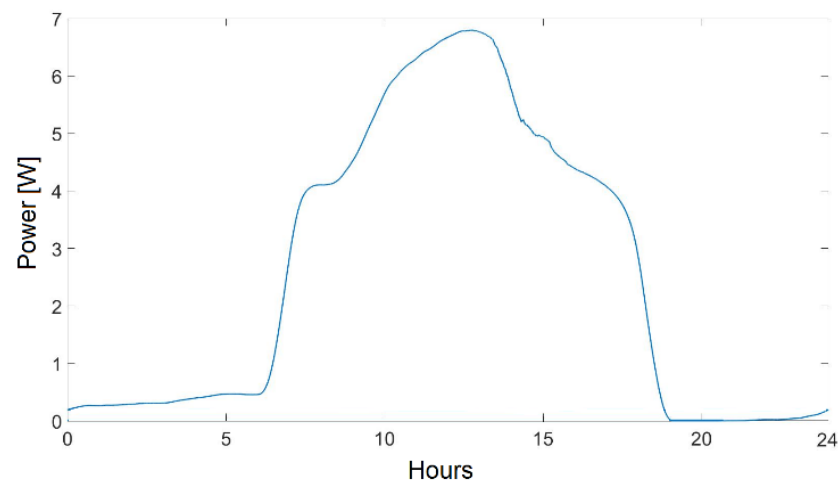

Figure 12: Power delivered by solar cells, extrapolated from the filtered profile of the light intensity shown in Figure 11.

A test was done in the Red Sea on a giant clam for valve gaping measurements. Figure 12 shows the valve gaping over a duration of 3 hours and 20 minutes.

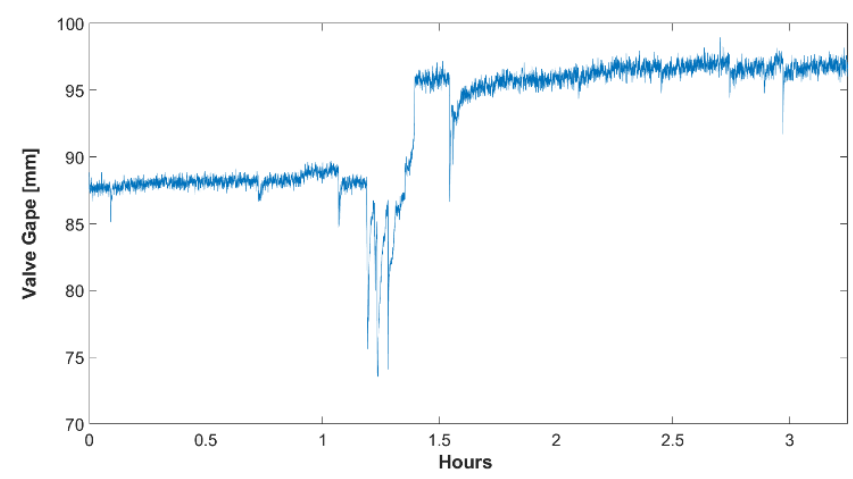

Figure 13: Valve gape measurement of a giant clam in the Red Sea.

\section{CONCLUSION}

With an ever increasing human impact on marine environments, monitoring environmental parameters and the behaviour of fauna in natural settings, as well as the changes resulting from human interference, has become of significant interest. Current technologies do not allow for remote, cost effective and high-resolution monitoring of data.

This has been adressed by creating a solar powered sensor buoy that consists of a primary module at the surface, for communication and power supply, that connects to a submerged secondary module, which acts as a hub for a variety of sensors. The data is parsed and relayed to the primary module and transmitted continuously to the internet using a cellular mobile network. The sensor buoy is a selfsufficient, compact, flexible and cheap solution to collect data in real time and allow the end user to access this in real time. As such, it will enable internet-of-things marine environmental monitoring and provide researchers with the ability to continuously monitor marine habitats with commercial or customized sensors.

\section{ACKNOWLEDGEMENTS}

This research was supported by King Abdullah University of Science and Technolgoy, the Red Sea Research Center and the CAASE project.

\section{REFERENCES}

[1] C. Mora, D. P. Tittensor, S. Adl, A. G. Simpson, and B. Worm, "How many species are there on Earth and in the ocean?," PLoS biology, vol. 9, no. 8, p. e1001127, 2011.

[2] D. Northcott, J. Sevadjian, D. A. Sancho-Gallegos, C. Wahl, J. Friederich, and F. P. Chavez, "Impacts of urban carbon dioxide emissions on sea-air flux and ocean acidification in nearshore waters," PloS one, vol. 14, no. 3, p. e0214403, 2019.

[3] S. Sendra, L. Parra, J. Lloret, and J. M. Jiménez, "Oceanographic multisensor buoy based on low cost sensors for Posidonia meadows monitoring in Mediterranean Sea," Journal of Sensors, vol. 2015, 2015.

[4] Y. Kaneda et al., "Dense ocean floor network system for mega thrust earthquakes and tsunamis (DONET) - towards understanding mega thrust earthquakes. SubOptic," SubOptic 2010, 2010.

[5] L. Liu et al., "A Seabed Real-Time Sensing System for In-Situ Long-Term Multi-Parameter Observation Applications," Sensors, vol. 19, no. 5, p. $1255,2019$.

[6] "Iridium contract costs." https://docs.rockblock.rock7.com/docs/iridiumcontract-costs (accessed Mar. 23, 2019.

[7] J. K. Pearman et al., "Cross-shelf investigation of coral reef cryptic benthic organisms reveals diversity patterns of the hidden majority," Scientific reports, vol. 8, no. 1, p. 8090, 2018.

[8] A. Kaidarova et al., "Tunable, Flexible Composite Magnets for Marine Monitoring Applications," Advanced Engineering Materials, vol. 20, no. 9, p. 1800229, 2018.

[9] A. Kaidarova et al., "Wearable Multifunctional Printed Graphene Sensors," npj Flexible Electronics, vol. 3, no. 1, pp. 1-10, 2019.

[10] A. Kaidarova, M. Marengo, G. Marinaro, N. Geraldi, C. M. Duarte, and J. Kosel, "Flexible and Biofouling Independent Salinity Sensor," Advanced Materials Interfaces, vol. 5, no. 23, p. 1801110 , 2018.

[11] N. Saeed, A. Celik, T. Al-Naffouri, and M.-S. Alouini, "Energy harvesting hybrid acoustic-optical underwater wireless sensor networks localization," Sensors, vol. 18, no. 1, p. 51, 2018. 
[12] S. F. Shaikh and M. M. Hussain, "Marine IoT: non-invasive wearable multisensory platform for oceanic environment monitoring," in 2019 IEEE 5th World Forum on Internet of Things (WF-IoT), 15-18 April 2019 2019, pp. 309-312, doi: 10.1109/WF-IoT.2019.8767310.

[13] S. Chakkor, E. A. Cheikh, M. Baghouri, and A. Hajraoui, "Comparative performance analysis of wireless communication protocols for intelligent sensors and their applications," arXiv preprint arXiv:1409.6884, 2014.

[14] B. Vejlgaard, M. Lauridsen, H. Nguyen, I. Z. Kovács, P. Mogensen, and M. Sorensen, "Coverage and capacity analysis of sigfox, lora, gprs, and nbiot," in 2017 IEEE 85th vehicular technology conference (VTC Spring), 2017: IEEE, pp. 1-5.

[15] Microchip, "Stand-Alone System Load Sharing and Li-Ion / Li-Polymer Battery Charge Management Controller," MCP73871 datasheet. [Online]. Available: https://cdnshop.adafruit.com/datasheets/MCP73871.pdf

[16] C. Schwartzmann, G. Durrieu, M. Sow, P. Ciret, C. E. Lazareth, and J.-C. Massabuau, "In situ giant clam growth rate behavior in relation to temperature: A one - year coupled study of high frequency noninvasive valvometry and sclerochronology," Limnology and oceanography, vol. 56, no. 5, pp. 1940-1951, 2011.
[17] S. Rossbach, V. Saderne, A. Anton Gamazo, and C. M. Duarte, "Light-dependent calcification in Red Sea giant clam Tridacna maxima," 2019.

[18] P. Soo and P. A. Todd, "The behaviour of giant clams (Bivalvia: Cardiidae: Tridacninae)," Marine biology, vol. 161, no. 12, pp. 2699-2717, 2014.

\section{CONTACT}

Prof. Jurgen Kosel, jurgen.kosel@kaust.edu.sa Mr Alexander Przybysz, alexander.przybysz@kaust.edu.sa 